\title{
Knockout of insulin and IGF-1 receptors on vascular endothelial cells protects against retinal neovascularization
}

\author{
Tatsuya Kondo, ${ }^{1}$ David Vicent, ${ }^{1}$ Kiyoshi Suzuma, ${ }^{1}$ Masashi Yanagisawa, ${ }^{2}$ George L. King, ${ }^{1}$ \\ Martin Holzenberger, ${ }^{3}$ and C. Ronald Kahn ${ }^{1}$
}

\begin{abstract}
${ }^{1}$ Research Division, Joslin Diabetes Center and Department of Medicine, Harvard Medical School, Boston, Massachusetts, USA

${ }^{2}$ Howard Hughes Medical Institute, University of Texas Southwestern Medical Center, Dallas, Texas, USA

${ }^{3}$ Institut National de la Santé et de la Recherche Médicale U515, Croissance, Différenciation et Processus Tumoraux, Hôpital Saint-Antoine, Paris, France
\end{abstract}

\begin{abstract}
Both insulin and IGF-1 have been implicated in control of retinal endothelial cell growth, neovascularization, and diabetic retinopathy. To precisely define the role of insulin and IGF-1 signaling in endothelium in these processes, we have used the oxygen-induced retinopathy model to study mice with a vascular endothelial cell-specific knockout of the insulin receptor (VENIRKO) or IGF-1 receptor (VENIFARKO). Following relative hypoxia, VENIRKO mice show a $57 \%$ decrease in retinal neovascularization as compared with controls. This is associated with a blunted rise in VEGF, eNOS, and endothelin-1. By contrast, VENIFARKO mice show only a 34\% reduction in neovascularization and a very modest reduction in mediator generation. These data indicate that both insulin and IGF-1 signaling in endothelium play a role in retinal neovascularization through the expression of vascular mediators, with the effect of insulin being most important in this process.
\end{abstract}

J. Clin. Invest. 111:1835-1842 (2003). doi:10.1172/JCI200317455.

\section{Introduction}

Diabetic retinopathy remains the leading cause of new blindness in adults in developed countries (1). The major causative factor for the development of diabetic retinopathy is hyperglycemia, which leads to increased vasopermeability, endothelial cell proliferation, and neovascularization. Diacylglycerol and PKC, which are increased and activated in the hyperglycemic condition, are known to be important in vascular cells to regulate permeability, cell growth, and angiogenesis in the diabetic state (2). These changes are associated with retinal ischemia, which is also a cause of the retinopathy of prematurity (ROP) (3-5). Indeed, diabetic retinopathy in humans and the experimental model of ROP in rodents are also similar with regard to overproduction of VEGF and other vascular factors (6-13) and their response

Received for publication November 22, 2002, and accepted in revised form March 19, 2003.

Address correspondence to: C. Ronald Kahn, Joslin Diabetes Center, One Joslin Place, Boston, Massachusetts 02215, USA. Phone: (617) 732-2635; Fax: (617) 732-2487;

E-mail: c.ronald.kahn@joslin.harvard.edu.

Conflict of interest: The authors have declared that no conflict of interest exists.

Nonstandard abbreviations used: retinopathy of prematurity (ROP); endothelin-1 (ET-1); vascular endothelial insulin receptor knockout (VENIRKO); vascular endothelial insulin-like growth factor receptor knockout (VENIFARKO); postnatal day (P); hypoxia-inducible factor- $1 \alpha$ (HIF-1 $\alpha$ ); periodic acid-Schiff (PAS); early growth response-1 (EGR-1). to treatments. Therefore, the ROP model has proven to be useful in defining the mechanisms and possible treatments of diabetic retinopathy (14).

Although the risk of progression in diabetic retinopathy over the long term is related to the degree of glycemic control (15), several clinical studies have demonstrated that intensive insulin therapy may cause a transient worsening of retinopathy in some individuals, even when compared to treatment with oral hypoglycemic agents (16-18). The biological mechanisms underlying the initial deterioration of diabetic retinopathy by intensive insulin therapy remain unknown; however, insulin signaling in endothelial cells has been shown to regulate the expression of some potential mediators of neovascularization, including $\operatorname{VEGF}(6,7)$, eNOS (8-10, 13), and endothelin-1 (ET-1) (11-13). IGF-1 also has been reported to be a potent stimulator of retinal endothelial cell growth and to play a major role in the development of diabetic retinopathy (19). Since there is extensive overlap and cross-talk between these two signaling systems, determining which is more important is difficult.

Using the Cre-loxP system, we have generated the vascular endothelial insulin receptor knockout (VENIRKO) mice (20), as well as mice with a vascular endothelial insulin-like growth factor receptor knockout (VENIFARKO). In the present study, we have investigated the role of insulin and IGF-1 signaling in endothelium and their regulation of VEGF, eNOS, and ET- 1 in retinopathy using the experimental ROP 
model in these mice. Our results indicate that, while both pathways may play a role in progression of retinal neovascularization through the activation of the angiogenesis factor, VEGF, and the vasoactive modulators, eNOS and ET-1, the insulin signaling seems to have a more important role and may produce a new therapeutic target in this disorder.

\section{Methods}

Animals. Endothelial cell-specific inactivation of the insulin receptor or IGF-1 receptor was achieved by cross-breeding Tie-2 Cre transgenic mice with mice carrying the floxed insulin receptor gene or IGF-1 receptor gene. As a result of the complex breeding, all of the mice have a mixed genetic background, including contributions from 129Sv, C57Bl/6, SJL, FVB, and DBA strains. None of these background strains carry genes for retinal degeneration, and no retinal degeneration was observed in any of the control mice. In all experiments, littermates from the same breeding pair were used as controls. All protocols for animal use and euthanasia were reviewed and approved by the Animal Care Committee of the Joslin Diabetes Center and were in accordance with NIH guidelines. Oxygen-induced retinopathy was induced in newborn mice according to the protocol of Smith et al. (14). On postnatal day 7 (P7), mice were placed, along with their dames, into a custom-built chamber in which the partial pressure of oxygen was raised to $75 \%$ and maintained for 5 days (P12), after which they were transferred back to cages in room air (normoxia). During this P12 to P17 period, mice, or at least their retinas, sense this change from hyperoxia to normoxia as a relative hypoxic condition, based on increased expression of hypoxia-inducible factor- $1 \alpha$ (HIF- $1 \alpha$ ). In this paper, we have referred to this as either the $\Delta \mathrm{O}_{2}$ model or relative hypoxia model.

Analysis of neovascularization. After the animals were anesthetized using pentobarbital $(25 \mathrm{mg} / \mathrm{kg})$ intraperitoneally, a median sternotomy was performed, and the pulsating left ventricle was identified. The left ventricle of the P17 mouse was perfused with $1 \mathrm{ml}$ of a $50 \mathrm{mg} / \mathrm{ml}$ solution of high-molecular-weight $(2,000 \mathrm{kDa})$ fluorescein-conjugated dextran (Sigma-Aldrich, St. Louis, Missouri, USA) in 10\% formalin as described previously (14). The eyes were then enucleated and placed in $10 \%$ formalin overnight. The retinas were dissected, radial cuts were made, and retinas were mounted on slides with VECTASHIELD (Vector Laboratories, Burlingame, California, USA). Coverslips were applied and sealed with clear nail polish. A fluorescent microscope (BX60; Olympus Optical Co., Tokyo, Japan) was used for visualization, and images were digitized using 3-CCD color video camera (HITACHI, Tokyo, Japan). In other mice, quantitation of neovascularization was performed using a technique described by Smith et al. (14), with minor modification. Briefly, the eyes were enucleated, placed in OCT (Sakura Finetek, Torrance, California, USA), and frozen at $-70^{\circ} \mathrm{C}$. Periodic acidSchiff-stained (PAS-stained) and hematoxylin-stained serial sections of $6-\mu \mathrm{m}$ thickness were examined in a masked fashion for the presence of neovascular buds projecting into the vitreous from the retina. The neovascularization score was defined as the mean number of neovascular nuclei per section found in 16 serial sections per eye. The mean score from these sections was used for statistical analysis of group differences.

Immunohistochemistry for endothelial mediators. VEGF, eNOS, ET-1, and DAPI, a nuclear marker, were visualized by immunohistochemical analysis in retinas. After a PBS wash, retinal frozen sections were incubated with $3 \%$ normal goat serum in $2.5 \%$ Triton X-100/PBS for 1 hour at room temperature. Incubations with primary Ab's to VEGF (Santa Cruz Biotechnology Inc., Santa Cruz, California, USA), eNOS (Becton Dickinson and Co., Franklin Lakes, New Jersey, USA), and ET-1 (Affinity BioReagents Inc., Golden, Colorado, USA) were performed in blocking solution ( $3 \%$ normal goat serum in $2.5 \%$ Triton X-100/PBS) overnight at $4^{\circ} \mathrm{C}$. After six washes with PBS, slides were incubated with appropriate secondary $\mathrm{Ab}$ 's conjugated to immunofluorescent dyes (Alexa 488 for green fluorescence and Alexa 546 for red fluorescence; Molecular Probes Inc., Eugene, Oregon, USA) in blocking solution for 2 hours at room temperature. After a rinse with PBS, slides were incubated with $100 \mathrm{ng} / \mathrm{ml}$ DAPI (Molecular Probes Inc.) for 15 minutes at room temperature. Sections were mounted with a Slow Fade kit (Molecular Probes Inc.) and then examined with a fluorescent microscope. The relative intensity of the immunoreaction products was graded blindly by two independent observers using the system described previously $(21,22)$. Results were expressed as the mean plus or minus SEM. ANOVA was used to compare the group differences.

\section{Results}

Physiological effects of insulin receptor or IGF-1 receptor inactivation in endothelial cells. At 2 months of age, body weight, fed and fasting glucose, fasting free fatty acid levels, and serum insulin concentrations were comparable in each control, VENIRKO, and VENIFARKO mouse (ref. 20; Table 1). Fasting triglyceride concentrations were reduced in VENIRKO mice approximately $20 \%(P<0.01)$ (ref. 20; and Table 1$)$. No abnormal or immature vascular development in VENIRKO and VENIFARKO animals was observed (data not shown). The development of retinal vascular network in controls, VENIRKO, and VENIFARKO mice was comparable (Figure 1, a and e, for VENIRKO control, others are not shown).

Prevention of retinal neovascularization in VENIRKO and VENIFARKO mice. To investigate if mice lacking insulin or IGF-1 receptors in endothelial cells were susceptible to relative hypoxia-induced retinal neovascularization (14), similar to that seen in ROP, control (lox/lox), VENIRKO, and VENIFARKO pups were exposed to $75 \%$ oxygen starting on P7 for 5 days, followed by a return to normoxia on P12 for an additional 5 days. The mice were then perfused using 
Table 1

Metabolic parameters of VENIRKO and VENIFARKO mice

\begin{tabular}{lcccc}
\hline & Control & VENIRKO & Control & VENIFARKO \\
Weight & $25.9 \pm 0.4$ & $26.3 \pm 0.5$ & $26.3 \pm 0.7$ & $24.4 \pm 0.6$ \\
Fasting glucose $(\mathrm{mg} / \mathrm{dl})$ & $59.3 \pm 1.9$ & $62.7 \pm 3.2$ & $61.2 \pm 2.6$ & $58.5 \pm 4.1$ \\
Random fed glucose $(\mathrm{mg} / \mathrm{dl})$ & $120 \pm 3$ & $123 \pm 4$ & $131 \pm 5$ & $125 \pm 3$ \\
Free fatty acids $(\mathrm{mEq} / \mathrm{l})$ & $1.39 \pm 0.03$ & $1.30 \pm 0.07$ & $1.21 \pm 0.07$ & $1.19 \pm 0.04$ \\
Triglycerides $(\mathrm{mg} / \mathrm{dl})$ & $160 \pm 8$ & $128 \pm 9^{A}$ & $35 \pm 7$ & $149 \pm 6$ \\
Insulin $(\mathrm{pg} / \mathrm{ml})$ & $317 \pm 35$ & $294 \pm 48$ & $364 \pm 28$ & $317 \pm 44$ \\
\hline
\end{tabular}

The number of mice in each group for 2 months old mice were: VENIRKO control $=41 ;$ VENIRKO $=30 ;$ VENIFARKO control $=20 ;$ VENIFARKO $=18$. AP $<0.05$ between VENIRKO control and VENIRKO. The data of VENIRKO control and VENIRKO were quoted from ref. 20.

fluorescein-conjugated dextran dissolved in $10 \%$ formalin, and the retinas were examined. In the $\Delta \mathrm{O}_{2}$ model, retinal flat-mount pictures of controls showed typical reactive central vaso-obliteration (avascular areas; Figure $1 \mathrm{~b}$, arrows), with formation of peripheral tufts that indicate newly formed vasculature (Figure 1f, arrowheads). By contrast, retinas of VENIRKO mice exposed to similar changes in oxygen tension exhibited more normal-appearing central vasculature and a lesser number of tufts as compared with retinas prepared from control animals (Figure 1, c and g). In VENIFARKO mice the extent of the avascular area and tuft formation was intermediate between control and VENIRKO retina (Figure 1, $\mathrm{d}$ and $\mathrm{h}$ ).

For further quantitative analysis, the nuclei of newly formed vessels that extend beyond the inner limiting membrane of the retina into the vitreous were counted using PAS- and hematoxylin-stained retinal frozen sections. In retinas of each control, VENIRKO, and VENIFARKO mouse maintained in normoxia, there were negligible numbers of nuclei protruding above the inner limiting membrane (Figure 2, a, b, e, and f). Sections from the two groups of control mice exposed to relative hypoxia had $50.0 \pm 2.7$ and $47.4 \pm 5.6$ neovascular nuclei (Figure 2, c and g, arrows; see Figure $6 a)$, whereas the number of neovascular nuclei in VENIRKO and VENIFARKO retina were significantly decreased to $21.6 \pm 5.7$ and $31.2 \pm 3.4$, respectively (Figure 2, $\mathrm{d}$ and h, arrows; see Figure 6a). Thus, the mean number of nuclei in VENIRKO and VENIFARKO mice in the $\Delta \mathrm{O}_{2}$ model was decreased $57 \%$ $(P<0.01)$ and $34 \%(P<0.05)$, respectively, from that observed in controls exposed to the same protocol (see Figure 6a). The number of endothelial nuclei extending beyond the inner limiting membrane in VENIRKO retina was also significantly reduced as compared with that in VENIFARKO retina $(P<0.05)$, and the difference in reduction between VENIRKO and VENIFARKO was $23 \%$.

Immunohistochemical analysis for vascular endothelial mediators. At least three vascular mediators, VEGF, eNOS, and ET-1, have been implicated in the pathogenesis of diabetic retinopathy and the ROP. To determine if these proteins were differentially regulated in control, VENIRKO, and VENIFARKO mice exposed to relative hypoxia, we assessed expression of VEGF,

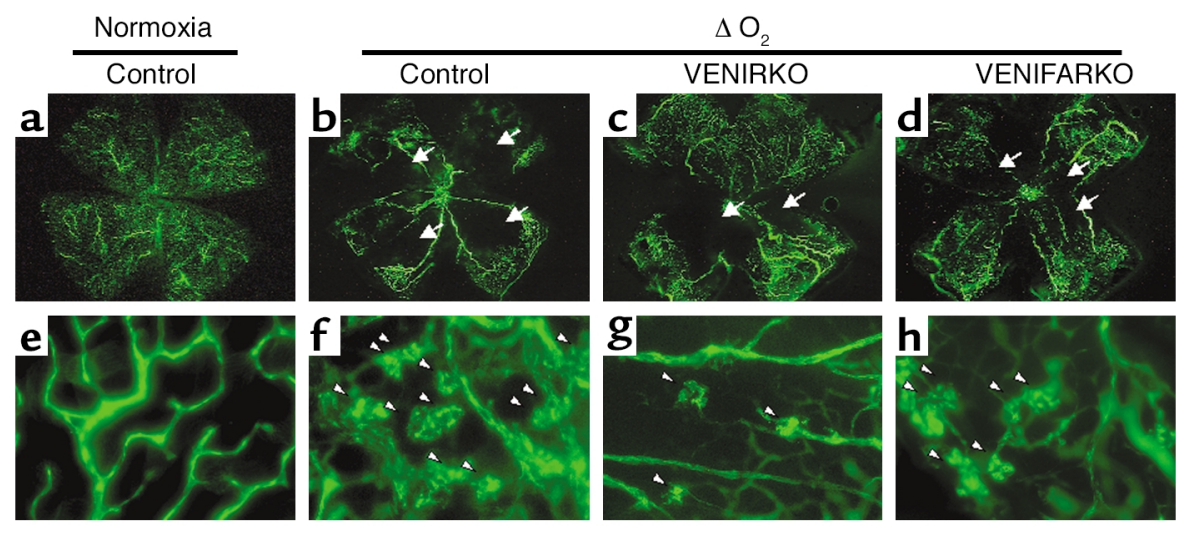

Figure 1

Retinal flat-mount pictures of VENIRKO and VENIFARKO mice exposed to hypoxia show decreased loss of blood vessels and fewer vascular tufts. Controls ( $\mathbf{b}$ and $\mathbf{f}$ ), VENIRKO ( $\mathbf{c}$ and $\mathbf{g}$ ), and VENIFARKO ( $\mathbf{d}$ and $\mathbf{h}$ ) mice were placed in a 75\% oxygen environment from P7 to P12, then returned to normoxia for 5 days. Control in normoxia sample is shown in a and e. Retinal vessels were visualized by fluorescein-conjugated dextran injection at P17. Control in normoxia shows no central avascular area (a) and no peripheral tuft formation (e). Low magnification of retina from control mice exposed to hypoxia (b) shows large central avascular areas (indicated by arrows). High magnifications of these retinas (f) reveal neovascular tuft formation (arrowheads). Retinas of VENIRKO mice (c and $\mathbf{g}$ ) show smaller avascular areas and a reduction of tufts. Retinas of VENIFARKO mice ( $\mathbf{d}$ and $\mathbf{h})$ show intermediate central avascular areas and tufts between control and VENIRKO mice. These flat-mount pictures are representative of at least six similar experiments. 


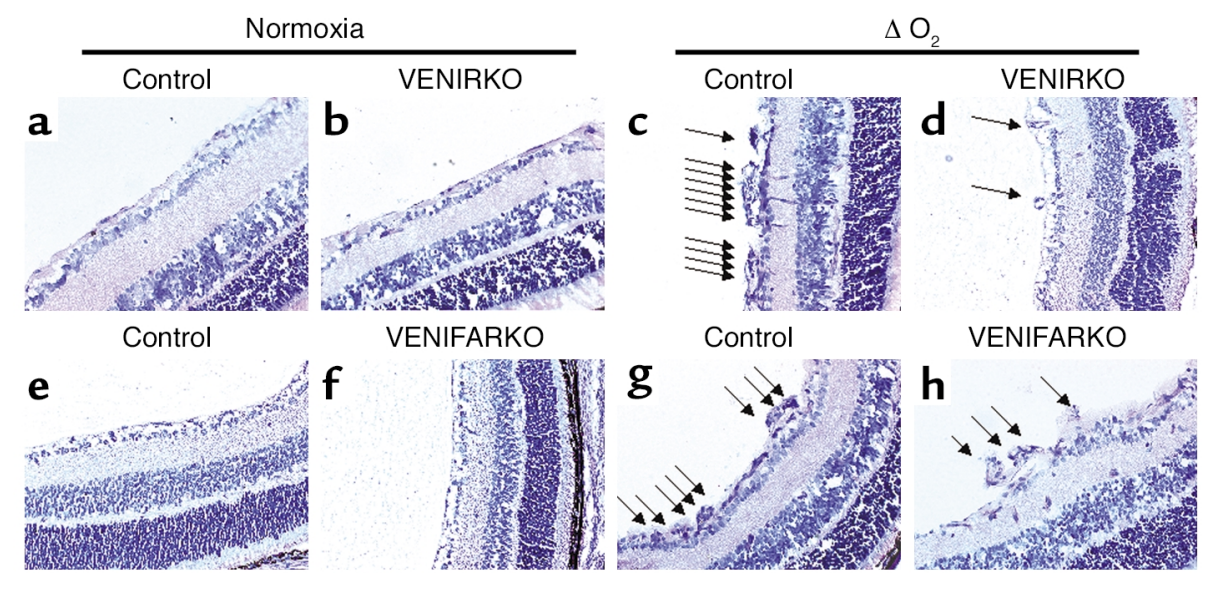

\section{Figure 2}

Retinas of VENIRKO and VENIFARKO mice exposed to hypoxia show fewer neovascular nuclei on PAS and hematoxylin staining. Frozen sections $(6 \mu \mathrm{m})$ of retinas were stained using PAS and hematoxylin. Retinas prepared from each control (a and e), VENIRKO (b), and VENIFARKO (f) mouse exposed in normoxia showed no neovascularization. Sections from each control mouse in the $\Delta \mathrm{O}_{2}$ model (c and g) had significant numbers of protruding nuclei above the inner limiting membrane, whereas the number of nuclei in VENIRKO retinas after the same treatment (d) was decreased $57 \%$ as compared with controls $(P<0.01$; see Figure $6 a)$. Furthermore, the number of nuclei in VENIFARKO retinas $(\mathbf{h})$ was reduced $34 \%$, as in control mice $(P<0.05$; see Figure 6 a).

eNOS, and ET-1 proteins in retinas using immunohistochemistry, with costaining of the nuclear marker DAPI. A stratum which consists of a few nucleus layers belongs to the ganglion cell layer, which locates the inner most retinal nuclei.

Under normoxia conditions, VEGF immunoreactivity was constitutively expressed in the vascular tissues of retina in each control, VENIRKO, and VENIFARKO mouse, in similar degree (Figure 3, a, b, e, and f). Following relative hypoxia, VEGF immunoreactivity was dramatically increased in the preretinal area (located above the nuclei of ganglion cell layer in DAPI-stained sections) in the controls following the change in oxygen tension (Figure 3, c and g). VEGF intensity in VENIRKO retina following relative hypoxia (Figure 3d) was significantly decreased 34\% (see Figure $6 \mathrm{~b} ; P<0.01$ ), and the reduction of VEGF intensity in VENIFARKO retina under relative hypoxia (Figure 3h) compared with control was not significant (18\%; see Figure 6b). The difference in VEGF intensity between the VENIRKO and VENIFARKO retinas was also statistically significant (see Figure $6 \mathrm{~b} ; P<0.05$ ).

Immunoreactive eNOS was also observed in normoxia retinas (Figure 4, a, b, e, and f). In control mice, following relative hypoxia, eNOS immunoreactivity increased fourfold (Figure 4, c and g; staining above the nuclei of ganglion cell layer). The intensity of eNOS in VENIRKO mice exposed to relative hypoxia (Figure 4d) also increased, but was $43 \%$ lower than that in control mice under the same conditions (see Figure $6 c ; P<0.01)$. The eNOS in the VENIFARKO mice after
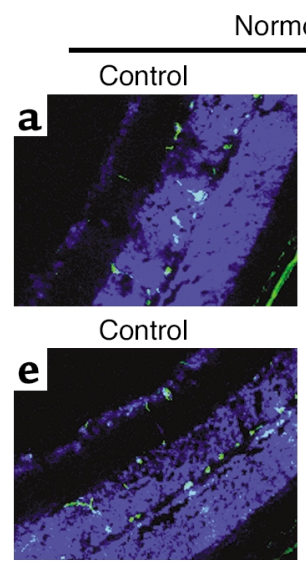

rmoxia
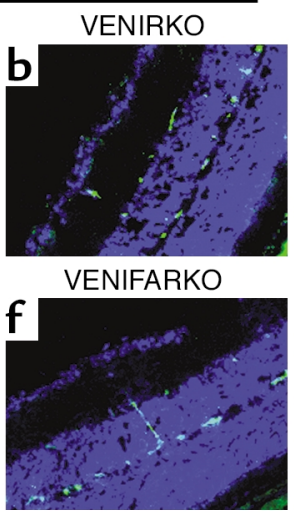

$\Delta \mathrm{O}_{2}$
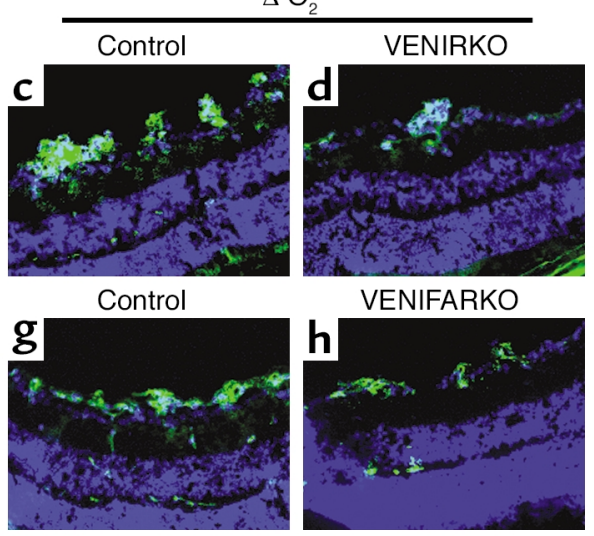

VENIFARKO

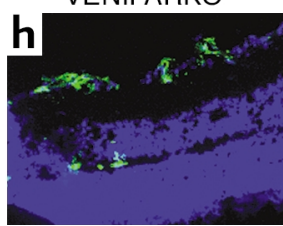

Figure 3

VEGF immunoreactivity in control retina is induced under hypoxia, but is less in VENIFARKO and even less in VENIRKO mice. VEGF immunoreactivity was constitutively observed in each control (a and e), VENIRKO (b), and VENIFARKO (f) retina and was highly induced in the $\Delta \mathrm{O}_{2}$ model of each control in the preretinal area ( $\mathbf{c}$ and $\left.\mathbf{g}\right)$. The induction of VEGF in VENIRKO retina $(\mathbf{d})$ was blunted $34 \%(P<0.01$; see Figure $6 \mathrm{~b}$ ), and that in VENIFARKO retina (h) was reduced $18 \%$ (not significant; see Figure $6 \mathrm{~b}$ ). 


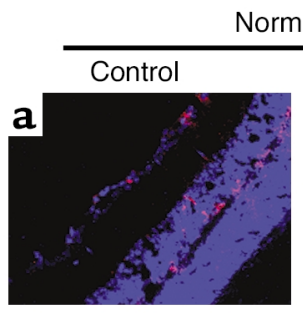

Control

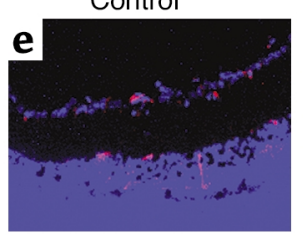

ormoxia

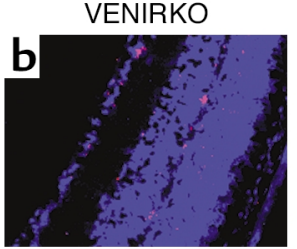

VENIFARKO

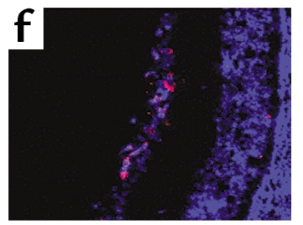

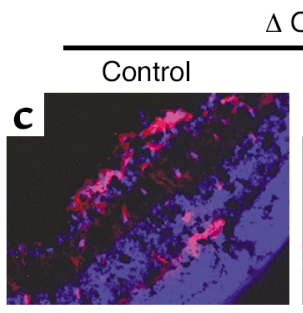

Control

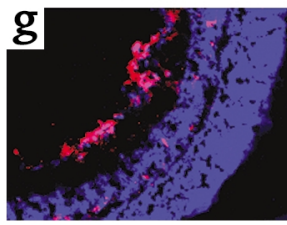

$\Delta \mathrm{O}_{2}$

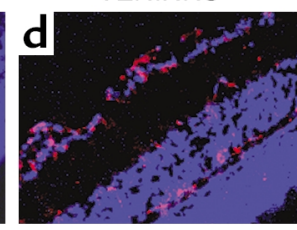

VENIFARKO

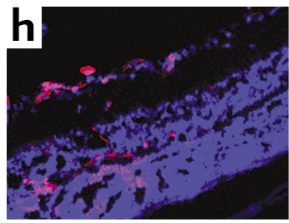

\section{Figure 4}

The eNOS immunoreactivity in control retina is increased under hypoxia, but is less in VENIFARKO and even less in VENIRKO mice. The eNOS protein was expressed in each control ( $\mathbf{a}$ and $\mathbf{e})$, VENIRKO $(\mathbf{b})$, and VENIFARKO (f) retina and was strongly increased when exposed to relative hypoxic conditions in the microvascular preretinal area in each control (c and $\mathbf{g})$. The induction of eNOS in VENIRKO retina (d) was decreased 43\% ( $P<0.01$; see Figure $6 \mathrm{c})$, and that in VENIFARKO retina $(\mathbf{h})$ was reduced $31 \%(P<0.05$; see Figure $6 \mathrm{c})$.

relative hypoxia (Figure $4 \mathrm{~h} ; P<0.05$ ) also increased, but was reduced $31 \%$ (see Figure $6 c ; P<0.05$ ) when compared with controls exposed to relative hypoxia.

ET-1 immunoreactivity under normoxic conditions was also constitutively detected in each control (Figure 5, a and e), VENIRKO (Figure 5b), and VENIFARKO (Figure 5f) mouse retinas. In control mice in the $\Delta \mathrm{O}_{2}$ model, ET-1 immunoreactivity increased over threefold in the microvascular preretinal region as compared with normoxic controls (Figure 5, c and g). ET-1 intensity in VENIRKO exposed to relative hypoxia (Figure $5 \mathrm{~d}$ ) was significantly lower, $37 \%$ when compared with controls (Figure $6 \mathrm{~d}, P<0.01$ ), and that in VENIFARKO was reduced by $24 \%$ under these same conditions (Figure $5 \mathrm{~h}$ and Figure $6 \mathrm{~d} ; P<0.05)$. Thus, the VENIRKO mice are more protected than VENIFARKO mice against the development of neovascular retinopathy, and this protection is related to a suppression of protein expression of VEGF, eNOS, and ET-1 in retinal endothelial cells following the relative hypoxia challenge.

\section{Discussion}

The pathogenesis of diabetic retinopathy is complex; while hyperglycemia is a major factor, diabetes is associated with changes not only in insulin and IGF-1, but also in many other hormones and metabolites, including free fatty acid, amino acids, advanced glycation end products, and components of the oxidative stress pathway. The development of diabetic retinopathy is also associated with retinal hypoxia leading to increased expression of endothelial mediators such as VEGF, eNOS, and ET-1 (6-13). Intensive insulin therapy to control hyperglycemia in diabetic patients is beneficial in the long term, but can promote a transient worsening of

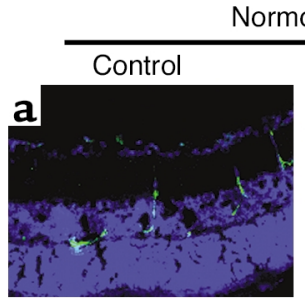

Normoxia

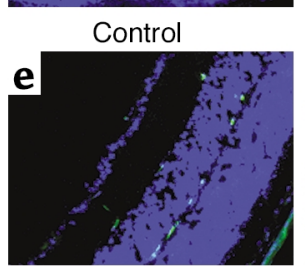

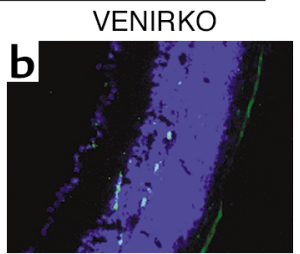

VENIFARKO

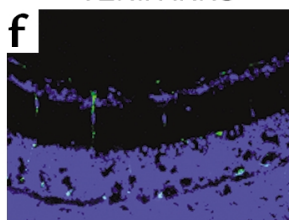

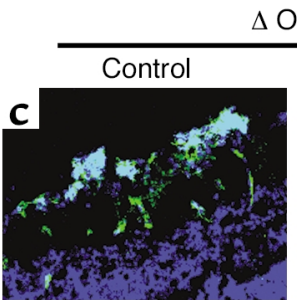

Control

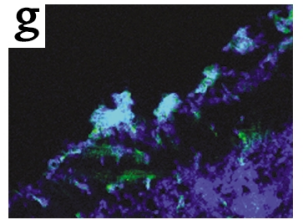

$\mathrm{O}$

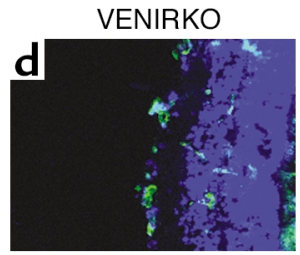

VENIFARKO

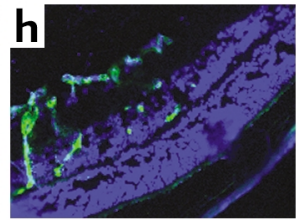

Figure 5

ET-1 immunoreactivity in control retina is upregulated under hypoxia, but is less in VENIFARKO and even less in VENIRKO mice. ET-1 immunoreactivity was constitutively seen in each control (a and $\mathbf{e}$ ), VENIRKO (b), and VENIFARKO (f) retina and was obviously upregulated in the $\Delta \mathrm{O}_{2}$ model of each control in the preretinal region (c and $\mathbf{g}$ ). The induction of ET-1 in VENIRKO retina (d) was blunted $37 \%$ $(P<0.01$; see Figure $6 \mathrm{~d})$, and that in VENIFARKO retina $(\mathbf{h})$ declined $24 \%(P<0.05$; see Figure $6 \mathrm{~d})$. 

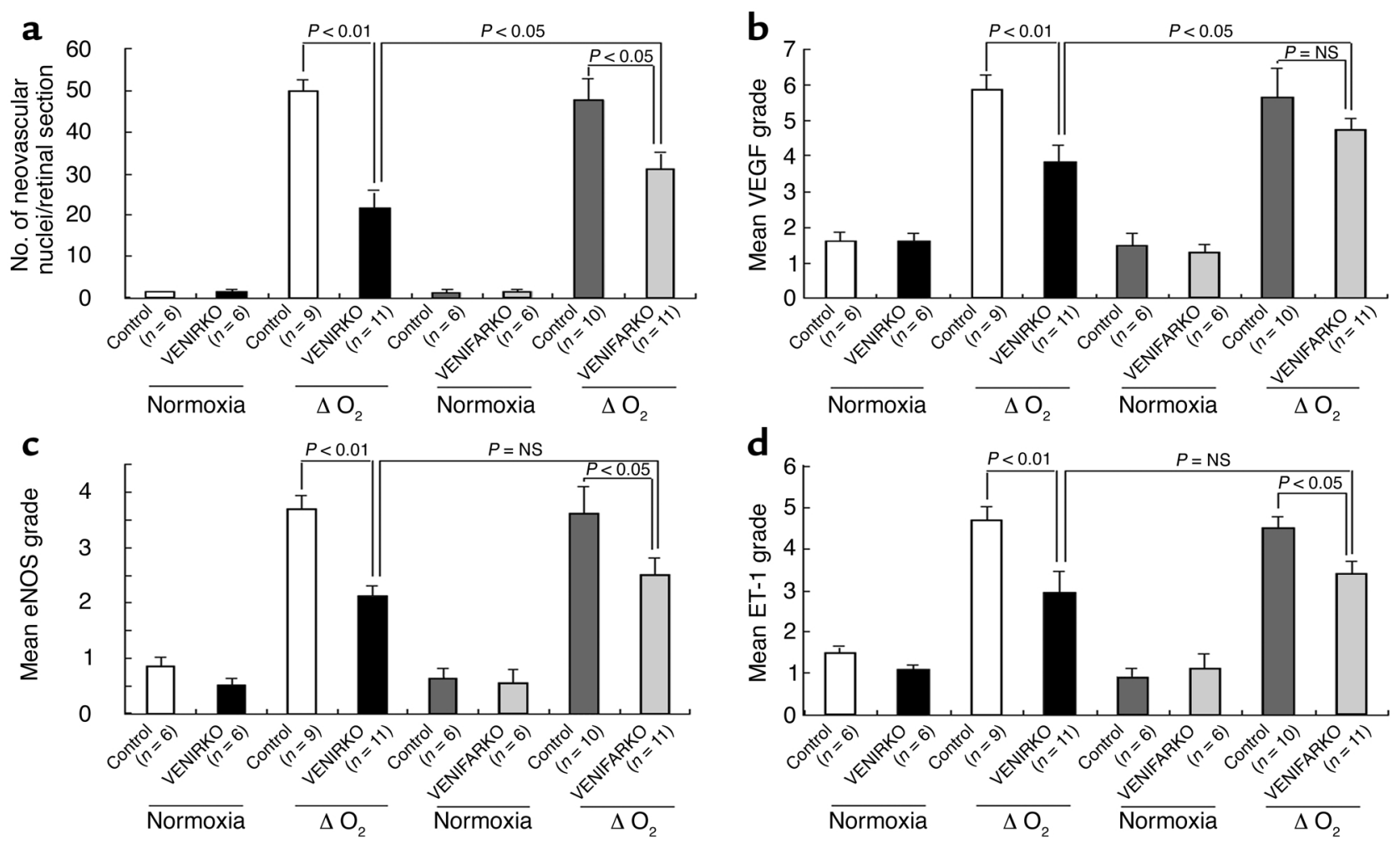

Figure 6

Quantitative results for suppression of neovascularization and VEGF, eNOS, and ET-1 immunoreactivity. (a) The number of neovascular nuclei per section is shown. Quantitative (b) VEGF, (c) eNOS, and (d) ET-1 immunoreactive intensity are shown.

retinopathy $(16,17,23,24)$. IGF-1 is homologous to insulin and acts through an independent but related receptor that shares many signaling components and cellular responses with the insulin receptor. IGF-1 has been reported to be associated with retinal endothelial cell growth and is considered to be a significant factor in development of diabetic retinopathy (19). In the present study, we demonstrate that disruption of the insulin or IGF-1 receptor in endothelial cells can reduce the vaso-obliteration and neovascularization process induced in neonatal mice exposed to relative hypoxic conditions, but the impact of reduction in the risk of retinopathy seems to be more prominent in insulin receptor knockout than IGF-1 receptor in endothelium.

In classical insulin-sensitive tissues, the insulin and IGF-1 receptor have been shown to regulate a wide variety of both metabolic and mitogenic functions. In general, insulin appears to preferentially activate the IRS/PI3K pathway to modulate metabolic effects, while the IGF-1 receptor appears to mediate more effective phosphorylation of Shc, its association with Grb2, and consequent activation of the MAPK pathway $(25,26)$. In previous studies, we have observed no significant differences in glucose metabolism among VENIRKO, VENIFARKO, and control mice under normal conditions (ref. 20; Table 1). On the other hand, the present investigation clearly shows that the insulin receptor and, to a lesser extent, the IGF-1 receptor in endothelial cells are involved in the proliferative response to relative hypoxia.
The growth of endothelial cells is regulated in large part by the expression of mediators derived from the endothelial cell itself. VEGF is a potent stimulator of both normal and abnormal vascular growth (27-29). Specific inhibition of VEGF activity prevents neovascularization in animal models of ROP (30). The proliferative effect of hyperglycemia on vascular endothelial cells is thought to be mediated by VEGF (31). It is known that hypoxia mimics the diabetic condition in many cells, including endothelium, as measured by induction of HIF- $1 \alpha$ and early growth response- 1 (EGR-1) expression (32). HIF-1 $\alpha$ and HIF-1 $\beta$ enhance the expression of erythropoietin and VEGF (33), while EGR-1 upregulates the transcription of $\mathrm{PKC} \alpha$ and PKC $\beta$ and activates Raf and MEK, leading to activation of the MAPKs, ERK-1 and -2, and enhancement of the cell growth of endothelium $(34,35)$. More recently, it has been reported that insulin stimulates diabetic blood-retinal barrier breakdown through induction of HIF-1 $\alpha$ and VEGF (7). NO is an important modulator of blood pressure due to its vasodilatory capability (36, 37) and plays a critical role in VEGF-induced angiogenesis and vascular hyperpermeability (38). In addition, several studies have demonstrated that $\mathrm{NO}$ is involved in modulation of cell proliferation, differentiation, and apoptosis in the vasculature (39). Furthermore, there is increasing evidence that NO-derived oxidants, such as peroxynitrite, might be important in situ mediators of oxidative injury in experimental models 
of retinal ischemia (40). Deficiency of eNOS, either through gene disruption or pharmacologic inhibition, significantly protects the developing retina from oxygen-induced retinopathy (41). ET-1 mRNA expression is also upregulated under the exposure of hypoxia (42), and ET-1 enhances VEGF-induced angiogenic-related effects on endothelial cells in vitro (43). Indeed, the vasoconstrictive response to hyperoxia has been proposed to be ET-1 dependent $(44,45)$.

Previous studies have shown that insulin and IGF-1 stimulate the expression of VEGF $(6,7)$, eNOS $(8-10$, $13)$, and ET-1 (11-13). Reduction in retinal neovascularization following disruption of insulin receptor in endothelial cells, which is accompanied by suppression of these vascular mediators, further implicates the role of these endothelial mediators in development of retinopathy. Another potential mechanism for prevention of neovascularization in these mice is loss of trophic support by PI3K/Akt due to impaired insulin or IGF-1 antiapoptotic signal in endothelial cells. It is well known that both insulin and IGF-1 have an ability to protect endothelial cells from apoptosis through the PI3K/Akt pathway, and suppression of endothelial cell apoptosis is associated with angiogenesis (46). In VENIRKO and VENIFARKO animals, deletion of insulin receptor or IGF-1 receptor in endothelial cells leads to less phosphorylation of Akt, which might increase apoptosis in those cells, thus reducing the neovascular formation response to relative hypoxia. The combined loss of cell protection and induction of vascular mediators could both contribute to this process.

Several clinical studies have demonstrated that intensive insulin therapy of diabetes causes a transient worsening of retinopathy $(16,17)$, though the incidence of intensive insulin therapy-induced retinopathy worsening is relatively small (47). Consistent with this, in this study we demonstrate that the blockade of the insulinsignaling pathway on the development of retinopathy in VENIRKO mice is only partial. This moderate reduction would allow for only a minor incidence of intensive insulin therapy-induced retinopathy.

Several investigators have suggested that IGF-1 signaling may be involved in this process, since IGF-1 levels rise after rapid control of hyperglycemia with insulin (48). Furthermore, systemic administration of an IGF-1 receptor antagonist can reduce the development of retinopathy in mice (19). The protective effect against development of retinopathy in VENIRKO mice was more than a $50 \%$ reduction as estimated by several scoring systems. VENIFARKO mice also had a reduction, but it was milder in VENIRKO mice (34\%). Thus, insulin signaling in endothelial cells appears to be more important than IGF-1 signaling in retinal endothelial cell proliferation, though the difference in suppression of retinopathy between VENIRKO and VENIFARKO mice is small. Furthermore, insulin and IGF-1 roles in retinopathy could involve cells other than the endothelium. Thus, both insulin and IGF-1 remain important factors in proliferation of retinal endothelial cells.
Recently, several other inhibitors, drugs, and mice deficient in expression of specific signaling molecules also have been found to have beneficial effects on the progression of the experimental ROP model. This raises the question as to whether insulin and/or IGF-1 receptor inhibition in endothelial cells or locally in the eye might be useful as a clinical treatment of diabetic retinopathy or ROP. In terms of clinical applicability, it is important to note that we have not observed any alterations in retinal structure or vasculature in any tissue in the VENIRKO and VENIFARKO mice. Thus, drug- or genebased therapies that inhibit insulin and/or IGF-1 receptor function in endothelial cells in the eye could provide a treatment for diabetic patients with retinopathy or premature infants exposed to hyperoxia. Further studies will be necessary to better define the molecular mechanisms of the development of retinopathy in relative hypoxia in both VENIRKO and VENIFARKO mice in response to diabetes, as well as in other retinal disorders.

\section{Acknowledgments}

The authors gratefully thank Izumi Suzuma for help in the morphological studies of retinal microvasculature. We appreciate Ravi Rauniyar and Lucy Goddard for technical assistance and Julie Marr for secretarial assistance in the preparation of the manuscript. This work was supported by a grant from the Manpei Suzuki Diabetes Foundation of Japan; NIH grants DK-31036, DK-33201, and DK-53105; and the Joslin Diabetes and Endocrinology Research Center grant.

1. Moss, S.E., Klein, R., and Klein, B.E. 1998. The 14-year incidence of visual loss in a diabetic population. Ophthalmology. 105:998-1003.

2. Koya, D., and King, G.L. 1998. Protein kinase C activation and the development of diabetic complications. Diabetes. 47:859-866.

3. Katzman, G.H. 1996. Retinopathy of prematurity: is suppression of neovascularization achievable? J. Pediatr. 129:618-619.

4. Aiello, L.P., et al. 1998. Diabetic retinopathy. Diabetes Care. 21:143-156.

5. Hamanaka, T., Akabane, N., Yajima, T., Takahashi, T., and Tanabe, A. 2001. Retinal ischemia and angle neovascularization in proliferative diabetic retinopathy. Am. J. Ophthalmol. 132:648-658.

6. Lu, M., et al. 1999. Insulin-induced vascular endothelial growth factor expression in retina. Invest. Ophthalmol. Vis. Sci. 40:3281-3286.

7. Poulaki, V., et al. 2002. Acute intensive insulin therapy exacerbates diabetic blood-retinal barrier breakdown via hypoxia-inducible factor- $1 \alpha$ and VEGF. J. Clin. Invest. 109:805-815. doi:10.1172/JCI200213776.

8. Jozkowicz, A., et al. 1999. Nitric oxide mediates the mitogenic effects of insulin and vascular endothelial growth factor but not of leptin in endothelial cells. Acta Biochim. Pol. 46:703-715.

9. Ding, Y., Vaziri, N.D., Coulson, R., Kamanna, V.S., and Roh, D.D. 2000. Effects of simulated hyperglycemia, insulin, and glucagon on endothelial nitric oxide synthase expression. Am. J. Physiol. Endocrinol. Metab. 279:E11-E17.

10. Kuboki, K., et al. 2000. Regulation of endothelial constitutive nitric oxide synthase gene expression in endothelial cells and in vivo: a specific vascular action of insulin. Circulation. 101:676-681.

11. Oliver, F.J., et al. 1991. Stimulation of endothelin-1 gene expression by insulin in endothelial cells. J. Biol. Chem. 266:23251-23256.

12. Desideri, G., Ferri, C., Bellini, C., De Mattia, G., and Santucci, A. 1997. Effects of ACE inhibition on spontaneous and insulin-stimulated endothelin-1 secretion: in vitro and in vivo studies. Diabetes. 46:81-86.

13. Cardillo, C., et al. 1999. Insulin stimulates both endothelin and nitric oxide activity in the human forearm. Circulation. 100:820-825.

14. Smith, L.E., et al. 1994. Oxygen-induced retinopathy in the mouse. Invest. Ophthalmol. Vis. Sci. 35:101-111.

15. Henricsson, M., Nilsson, A., Janzon, L., and Groop, L. 1997. The effect of glycaemic control and the introduction of insulin therapy on retinopathy in non-insulin-dependent diabetes mellitus. Diabet. Med. 14:123-131.

16. Lauritzen, T., Frost-Larsen, K., Larsen, H.W., and Deckert, T. 1985. 
Two-year experience with continuous subcutaneous insulin infusion in relation to retinopathy and neuropathy. Diabetes. 3(Suppl.):74-79.

17. Dahl-Jorgensen, K., Brinchmann-Hansen, O., Hanssen, K.F., Sandvik, L. and Aagenaes, O. 1985. Rapid tightening of blood glucose control leads to transient deterioration of retinopathy in insulin dependent diabetes mellitus: the Oslo study. Br. Med. J. (Clin. Res. Ed.) 290:811-815.

18. Roysarkar, T.K., Gupta, A., Dash, R.J., and Dogra, M.R. 1993. Effect of insulin therapy on progression of retinopathy in noninsulin-dependent diabetes mellitus. Am. J. Ophthalmol. 115:569-574.

19. Smith, L.E., et al. 1999. Regulation of vascular endothelial growth factor-dependent retinal neovascularization by insulin-like growth factor1 receptor. Nat. Med. 5:1390-1395.

20. Vicent, D., et al. 2003. The role of endothelial insulin signaling in the regulation of vascular tone and insulin resistance. J. Clin. Invest. 111:1373-1380. doi:10.1172/JCI200315211.

21. Page, C., Rose, M., Yacoub, M., and Pigott, R. 1992. Antigenic heterogeneity of vascular endothelium. Am. J. Pathol. 141:673-683.

22. Lutty, G.A., McLeod, D.S., Merges, C., Diggs, A., and Plouet, J. 1996. Localization of vascular endothelial growth factor in human retina and choroid. Arch. Ophthalmol. 114:971-977.

23. Cunha-Vaz, J. 1998. Lowering the risk of visual impairment and blindness. Diabet. Med. 4(Suppl.):S47-S50.

24. Shichiri, M., Kishikawa, H., Ohkubo, Y., and Wake, N. 2000. Long-term results of the Kumamoto Study on optimal diabetes control in type 2 diabetic patients. Diabetes Care. 2(Suppl.):B21-B29.

25. Cheatham, B., et al.1994. Phosphatidylinositol 3-kinase activation is required for insulin stimulation of Pp70 S6 kinase, DNA synthesis, and glucose transporter translocation. Mol. Cell Biol. 14:4902-4911.

26. Mendez, R., Myers, M.G., Jr., White, M.F., and Rhoads, R.E. 1996. Stimulation of protein synthesis, eukaryotic translation initiation factor 4E phosphorylation, and PHAS-I phosphorylation by insulin requires insulin receptor substrate 1 and phosphatidylinositol 3-kinase. Mol. Cell Biol. 16:2857-2864.

27. Aiello, L.P., et al. 1994. Vascular endothelial growth factor in ocular fluid of patients with diabetic retinopathy and other retinal disorders. N. Engl. J. Med. 331:1480-1487.

28. Miller, J.W., Adamis, A.P., and Aiello, L.P. 1997. Vascular endothelia growth factor in ocular neovascularization and proliferative diabetic retinopathy. Diabetes Metab. Rev. 13:37-50.

29. Simpson, D.A., et al. 1999. Expression of the VEGF gene family during retinal vaso-obliteration and hypoxia. Biochem. Biophys. Res. Commun. 262:333-340.

30. Ozaki, H., et al. 2000. Blockade of vascular endothelial cell growth factor receptor signaling is sufficient to completely prevent retinal neovascularization. Am. J. Pathol. 156:697-707.

31. Stone, J., and Maslim, J. 1997. Mechanisms of retinal angiogenesis. Prog. Retin. Eye Res. 16:157-181.

32. Ten, V.S., and Pinsky, D.J. 2002. Endothelial response to hypoxia: physiologic adaptation and pathologic dysfunction. Curr. Opin. Crit. Care. 8:242-250.

33. Semenza, G.L. 2000. HIF-1: mediator of physiological and pathophysiological responses to hypoxia. J. Appl. Physiol. 88:1474-1480.

34. Yan, S.F., et al. 1999. Hypoxia-associated induction of early growth response-1 gene expression. J. Biol. Chem. 274:15030-15040.

35. Lo, L.W., et al. 2001. Endothelial exposure to hypoxia induces Egr-1 expression involving PKCalpha-mediated Ras/Raf-1/ERK1/2 pathway. J. Cell Physiol. 188:304-312.

36. Rees, D.D., Palmer, R.M., and Moncada, S. 1989. Role of endotheliumderived nitric oxide in the regulation of blood pressure. Proc. Natl. Acad. Sci.U. S. A. 86:3375-3378.

37. Murad, F., et al. 1992. The nitric oxide-cyclic GMP signal transduction pathway in vascular smooth muscle preparations and other tissues. Jpn. J. Pharmacol. 58(Suppl.):150P-157P.

38. Fukumura, D., et al. 2001. Predominant role of endothelial nitric oxide synthase in vascular endothelial growth factor-induced angiogenesis and vascular permeability. Proc. Natl. Acad. Sci. U. S. A. 98:2604-2609.

39. Ono, Y., Ono, H., Matsuoka, H., Fujimori, T., and Frohlich, E.D. 1999 Apoptosis, coronary arterial remodeling, and myocardial infarction after nitric oxide inhibition in SHR. Hypertension. 34:609-616.

40. Adachi, K., et al. 1998. Inhibition of NMDA receptors and nitric oxide synthase reduces ischemic injury of the retina. Eur. J. Pharmacol. 350:53-57.

41. Brooks, S.E., et al. 2001. Reduced severity of oxygen-induced retinopathy in eNOS-deficient mice. Invest. Ophthalmol. Vis. Sci. 42:222-228.

42. Tadesse, M., Yan, Y., Yossuck, P., and Higgins, R.D. 2001. Captopril improves retinal neovascularization via endothelin-1. Invest. Ophthalmol. Vis. Sci. 42:1867-1872.

43. Salani, D., et al. 2000. Endothelin-1 induces an angiogenic phenotype in cultured endothelial cells and stimulates neovascularization in vivo. Am. J. Pathol. 157:1703-1711.

44. McLeod, D.S., Brownstein, R., and Lutty, G.A. 1996. Vaso-obliteration in the canine model of oxygen-induced retinopathy. Invest. Ophthalmol. Vis. Sci. 37:300-311.

45. Takagi, C., et al. 1996. Endothelin-1 action via endothelin receptors is a primary mechanism modulating retinal circulatory response to hyperoxia. Invest. Ophthalmol. Vis. Sci. 37:2099-2109.

46. Hermann, C., Assmus, B., Urbich, C., Zeiher, A.M., and Dimmeler, S 2000. Insulin-mediated stimulation of protein kinase Akt: a potent survival signaling cascade for endothelial cells. Arterioscler. Thromb. Vasc. Biol. 20:402-409.

47. 1998. Early worsening of diabetic retinopathy in the Diabetes Control and Complications Trial. Arch. Ophthalmol. 116:874-886.

48. Attia, N., et al. 1999. Changes in free insulin-like growth factor-1 and leptin concentrations during acute metabolic decompensation in insulin withdrawn patients with type 1 diabetes. J. Clin. Endocrinol. Metab. 84:2324-2328. 